Journal of Engineering and Applied Sciences 14 (16): 5616-5626, 2019

ISSN: $1816-949 \mathrm{X}$

(C) Medwell Journals, 2019

\title{
Synthesis of Medium and Long-Chain Structured Lipids from Oil Blend of Palm Olein and Palm Kernel Olein by Geobacillus sp. Strain ARM Lipase
}

\author{
${ }^{1,2}$ Raja Noor Zaliha Raja Abd. Rahman, ${ }^{1}$ Mohamad Firdaus Md Yusof and \\ ${ }^{1,3}$ Nor Hafizah Ahmad Kamarudin \\ ${ }^{1}$ Enzyme and Microbial Technology Research Centre, Universiti Putra Malaysia, \\ 43400 Serdang, Selangor, Malaysia. \\ ${ }^{2}$ Department of Microbiology, Faculty of Biotechnology and Biomolecular Sciences, \\ Universiti Putra Malaysia, 43400 Serdang, Selangor, Malaysia \\ ${ }^{3}$ Centre of Foundation Studies of Agricultural Sciences, Universiti Putra Malaysia, \\ 43400 Serdang, Selangor, Malaysia \\ hafizah_kamar@upm.edu.my,+603-89466934,+603-8946 6997
}

\begin{abstract}
Development of Structured Lipid (SL) a new generation of lipids with high nutritional and health value remains an interesting area in functional food processing. Design of new SL by enzymatic modification has revealed many interesting findings and the search for more potent biocatalyst is an urgent need to fulfill the consumers demand. In this research, we aimed to investigate and evaluate the ability of sn-1, 3 regioselective thermostable lipase from locally isolated Geobacillus sp. strain (designated as ARM lipase) in catalyzing the transesterification of Palm Olein (POo) and Palm Kernel Olein (PKO) a source rich in oleic, palmitic and lauric acid. Study conducted showed that the chemical composition of oil blend POo: PKO particularly the Fatty Acid (FA) chain length and distribution of FA on the glycerol backbone was altered, resulting in the production of Medium and Long Chain length of acylglycerol(MLCT) and Diacylglycerol (DAG). Two important parameters were investigated, reaction time and temperature. Both time and temperature of reaction have direct influence on the conversion of $\mathrm{POo}: \mathrm{PKO}$ into $\mathrm{MLCT}$ and DAG which increases with an increase in time and temperature up to $10 \mathrm{~h}$ and $55^{\circ} \mathrm{C}$, respectively. A gradual increment of MLCT and DAG was observed at $35-50^{\circ} \mathrm{C}$ and TAG reached its highest conversion with $12 \% \mathrm{MLCT}$ and $16 \% \mathrm{DAG}$ at $55^{\circ} \mathrm{C}$ after $10 \mathrm{hreaction}$. The result indicated the proficiency of ARM lipase to catalyze reaction at high temperature and remains stables for prolong reaction time. Analysis on the physical changes of the reacted oil blend showed that the oil blend has sharp melting profile and accelerated crystallization behavior. These attributes are critical for production of confectionery, margarine and shortening. ARM lipase demonstrates high competency as a new biocatalyst for lipid processing industry.
\end{abstract}

Key words: 1,3 regioselective, thermostable, lipase, medium and long chain structured lipids, reaction, ARM, margarine

\section{INTRODUCTION}

The potential biological function and nutritional perspective of Structured Lipids (SL) a new generation of healthy fats have primarily attracted health conscious consumers. The synthesis of low calorie lipid which is commonly categorized into several types Short-and Long Chain acyl residues (SLCTs), Medium-and Long Chain acyl residues (MLCTs) and Diacylglycerols (DAGs) has recently grow into an important area in oil processing industry. SL can be considered as an ideal lipid substitute only if it contains essential fatty acids, unsaturated fatty acids and gives no harmful effects to the consumers (Cao et al., 2013). The tailor-made SL constituting of Medium Chain Fatty Acid (MCFA) which can be developed by modification and restructuring the position and composition of fatty acids in its naturally occurring form is recognized to be safer and healthier as it can be easily hydrolyzed by lipases, absorbed into the intestines, hence, reducing the accumulation of body fats and problems associated with obesity (Wang et al., 2012). Additionally, incorporation of Long Chain Fatty Acid (LCFA) in SL provides health and nutritional benefits.

As consumers continuously demand for healthier food products, synthesizing SL from natural oils by lipase-mediated modification is economically feasible and more 'greener' compared to conventional chemical reaction using other high-cost substrates. Enzymatic transesterification, specifically lipase-catalyzed reaction prevails in many aspects over chemical reactions.

Corresponding Author: Nor Hafizah Ahmad Kamarudin, Centre of Foundation Studies of Agricultural Sciences, Universiti Putra Malaysia, 43400 Serdang, Selangor, Malaysia 
Enzyme-mediated catalysis reaction is known for high specificity with fewer side products and can be conducted at mild and moderate reaction conditions (Palla and Carrine, 2014).

Furthermore, the nature of lipase exhibiting high specific activity, substrate specificity and stability in extreme conditions such as temperature and organic solvents makes it a potent biocatalyst in lipid processing. Substrate specificity of lipases is one of the key criteria in lipid modification. Lipase with regioselective property acts only on primary ester bonds (1,3-specific lipases) and represents a good candidate for production of specific SLs (Palla and Carrin, 2014). For instance, sn-1, 3 specific lipase from Rhizomucor miehei was used in the production of SLs while the enzymatic synthesis of 1,3-dioleoyl-2-Palmitoylglycerol (OPO), one of the components of human milk fat was carried out using 1,3 specific Aspergillus oryzae lipase (Palla and Carrin, 2014; Cai et al., 2015). Enzymatic modification allows a selective incorporation of a desired acyl moiety onto a specific position of Triacylglycerides (TAG) (Lee et al., 2015).

While positional specificity is important to target certain fatty acids in triglycerides for restructuring the fatty acid position and composition, the limited number of 1,3 specific lipase available for synthesis of SLs has restricted the application of 1,3 specific lipase, hence, leading to a relatively expensive process. Development of new lipases for lipid modification is thus a never-ending quest in order to provide vast options and alleviate the production cost. Several reported lipases used in the synthesis of SL include Pseudomonas cepacia lipase for the production of 1,3-dioleoyl-2-Palmitoylglycerol(OPO) and immobilized lipase from Rhizomucor miehei, Novozymes for MLCT synthesis (Koh et al., 2011).

Another important factor in the bioconversion of lipids by lipase is the type of substrate used. Many SLs have been predominantly produced from vegetable oils such as canola, flaxseed and soybean oil (Wang et al., 2012; Khodadadi et al., 2013; Palla and Carrin, 2014). Oil palm, a major plantation crop in the country and many other tropical regions is a source of palm oil and palm kernel oil. Until recently, palm oil has been known as the highest edible oil worldwide. After fractionation, the liquid portion is known as palm olein and palm kernel olein, respectively. Palm oil which constituted of a balanced composition between saturated and unsaturated fatty acids namely palmitic (C16) and oleic acid (C18) contains high nutritional value and offers greater advantages compared to other vegetable oils. Palm kernel oil on the other hand, resembles coconut oil with high content of lauric acid (C12) and exhibits greater stability at high temperature and better shelf-life. A study conducted on modification of palm oil has succeeded to produce OPO an important infant formula (Chen et al., 2004).

\section{MATERIALS AND METHODS}

Bacterial sources: $E$. coli TOP10 harboring recombinant pTrcHis/ARM lipase in $80 \%$ glycerol stock was obtained from Enzyme and Microbial Technology Research Center, Universiti Putra Malaysia.

Preparation of crude enzyme: The bacterial culture was grown in $5 \mathrm{~L}$ Erlenmayer flask containing $1 \mathrm{~L}$ of LB broth medium supplemented with $50 \mu \mathrm{g} / \mathrm{mL}$ of ampicilin on a rotary shaker $(200 \mathrm{rpm})$ at $37^{\circ} \mathrm{C}$. The culture was induced with $1 \mathrm{mM} \mathrm{IPTG}$ (Isopropyl- $\beta$-D-thiogalactopyranoside) when optical density $O . D_{600 \mathrm{~nm}}$ reached approximately 0.5 and further incubated for $17 \mathrm{~h}$ at $37^{\circ} \mathrm{C}$. Following incubation, cells $(20 \mathrm{~mL})$ were harvested by centrifugation $\left(10,000 \mathrm{xg}, 10 \mathrm{~min}, 4^{\circ} \mathrm{C}\right)$ and the pellet was resuspended with $20 \mathrm{~mL}$ of $50 \mathrm{mM}$ Tris- $\mathrm{HCl} \mathrm{pH} 8.0$ before sonication (Branson 250 sonifier: 20 intermittent cycles, output 2, duty cycle 28-30) and cleared by centrifugation at $10,000 \mathrm{xg}$ for $10 \mathrm{~min}$ at $4^{\circ} \mathrm{C}$. The soluble fraction containing the crude lipase was analyzed for lipolytic activity prior to immobilization.

Lipase activity assay: Lipase activity assay was performed according to Kwon and Rhee (1986), calorimetric method with slight modification. Reaction mixture containing $10 \mu \mathrm{L}$ of crude lipase, $0.05 \mathrm{~g}$ of immobilized lipase, $2.5 \mathrm{~mL}$ of olive oil emulsion (1:1, $\mathrm{v} / \mathrm{v}), 20 \mu \mathrm{L}$ of $0.02 \mathrm{M} \mathrm{CaCl}_{2}$ and $990 \mu \mathrm{L}$ of $50 \mathrm{mM}$ Tris$\mathrm{HCl}$ buffer were incubated in a water bath shaker at an agitation rate of $200 \mathrm{rpm}$ for $30 \mathrm{~min}$ at $60^{\circ} \mathrm{C}$. The enzymatic reaction was terminated by adding $1 \mathrm{~mL}$ of $6 \mathrm{M} \mathrm{HCl}$ and $5 \mathrm{~mL}$ isooctane followed by vigorous vortexing. The upper layer of isooctane $(4 \mathrm{~mL})$ containing the liberated fatty acid was transferred into a test tube containing $1 \mathrm{~mL}$ copper-pyridine reagent for analysis. The amount of free fatty acid released was measured by the absorbance reading at $715 \mathrm{~nm}$. Oleic acid standard curve was used to determine the lipolytic activity. One unit of lipase activity is defined as the rate of $1 \mu$ mole fatty acid released per minute. The enzymatic assay was performed in triplicates.

Immobilization of crude ARM lipase: The crude lipase suspension was immobilized using simple adsorption technique onto different support matrices namely chitosan, amberlite of each support matrix was added to $10 \mathrm{~mL}$ crude enzyme solution (containing about 
$3000 \mathrm{U}$ ). To aid the adsorption process, the mixture was incubated in a water bath shaker at $20^{\circ} \mathrm{C}$ for $2 \mathrm{~h}$. The immobilized lipase was vacuum-filtered through whatman No. 1 filter paper and washed with $20 \mathrm{~mL}$ of distilled water to remove any non-adsorbed lipase. The washing process was repeated, until the collected supernatant contained no protein trace as measured by Bradford protein assay.

The dried immobilized lipase was stored in a $-80^{\circ} \mathrm{C}$ freezer prior to lyophilized using Christ Alpha 1-2 LD freeze dryer for $24 \mathrm{~h}$. The enzymatic activity of the immobilized lipase $(0.05 \mathrm{~g})$ was measured and stored in a $-20^{\circ} \mathrm{C}$ freezer before use (Rahman et al., 2008; Branco et al., 2010). The best support matrix for ARM lipase was chosen based on the highest activity performed by the immobilized lipase.

Enzymatic transesterification of palm olein and palm kernel oil: Enzymatic transesterification of the blend $\mathrm{RBD}$ POo and RBD PKO with mass (w/w) ratio 50:50 containing $50 \mathrm{~g}$ of total lipids was carried out in a solventfree media, following the method described by Lee et al. (2015) with slight modification. The blend was stirred at $350 \mathrm{rpm}$ and temperature was maintained with water bath. Five sets of oil blend were prepared for different temperatures $\left(35,40,45,50\right.$ and $\left.55^{\circ} \mathrm{C}\right)$. The reaction started with the addition of immobilized ARM lipase of $250 \mathrm{U}$ once the desired temperatures were achieved. Reacted samples were taken on every hour and further centrifuged and filtered with $0.45 \mu \mathrm{m}$ PTFE membrane filter to separate the enzyme. Samples were stored at $20^{\circ} \mathrm{C}$ for further analyses.

Determination of fatty acids composition: Preparation of Fatty Acids Methyl Ester (FAME) was carried out according to O'fallon et al. (2007) method. Oil sample $(20 \mu \mathrm{L}), 0.7 \mathrm{~mL} 10 \mathrm{~N} \mathrm{KOH}$ in water and $5.3 \mathrm{~mL}$ methanol were placed in $15 \mathrm{~mL}$ centrifuge tube. The tube was incubated at $55^{\circ} \mathrm{C}$ water bath for $1.5 \mathrm{~h}$ and vigorously shaken at every $20 \mathrm{~min}$. Following incubation, the mixture was left cooled to room temperature under cold tap water and $0.58 \mathrm{~mL}$ of $24 \mathrm{~N} \mathrm{H}_{2} \mathrm{SO}_{4}$ was added. Sample was then mixed by inversion until precipitated $\mathrm{K}_{2} \mathrm{SO}_{4}$ present. It was then incubated again for $1.5 \mathrm{~h}$ at $55^{\circ} \mathrm{C}$ water bath with $5 \mathrm{sec}$ hand shaking every $20 \mathrm{~min}$. Hexane (3 $\mathrm{mL}$ ) was added and vortexed for $5 \mathrm{~min}$. The hexane layer containing FAME was transferred into chromatography vial for Gas Chromatography (GC) analysis.

Analysis of the derivatizedFAME was conducted using Agilent (Santa Clara California, USA), GC A2890 equipped with Flame Ionization Detector (FID) (Lee et al., 2015). The capillary column was BPX70 70\% Cynopropyl Polysilphenylene-siloxane (SGE Analytical Science, Ridgewood Victoria, Australia), $30 \mathrm{~m}$ in length with internal diameter of $0.32 \mu \mathrm{m}$. The injector and detector temperature were maintained at 250 and $280^{\circ} \mathrm{C}$, respectively while the carrier gas was nitrogen. The split ratio was 15:1. Oven temperature was programmed as follows the column was held at $150^{\circ} \mathrm{C}$ for $0.5 \mathrm{~min}$ increased to $180^{\circ} \mathrm{C}$ at a rate of $10^{\circ} \mathrm{C} / \mathrm{min}, 220^{\circ} \mathrm{C}$ at a rate of $1.5^{\circ} \mathrm{C} / \mathrm{min}$ and $260^{\circ} \mathrm{C}$ at a rate of $30^{\circ} \mathrm{C} / \mathrm{min}$ and finally held at $260^{\circ} \mathrm{C}$ for $5 \mathrm{~min}$. Duplicate measurements for each sample was performed. Results were expressed as mean \pm standard deviation.

Determination of TAG composition: A waters HPLC e2695 (Milford Massachusetts, USA) equipped with Evaporative Light Scattering Detector (ELSD) was used to determine the TAG class composition. The separation of TAG was done following the method outlined by Lee et al. (2015). The column used was a pre-coated silica reversed phase C18 HPLC column, Lichro CART $5 \mu \mathrm{m}(4 \mathrm{~mm} \times 25 \mathrm{~cm})$ from Merck (Darmstadt, Germany). The gradient mobile phase was a mixture of acetone (A) and acetonitrile (B) while the flow rate was $1 \mathrm{~mL} / \mathrm{min}$ with a total run time of $55 \mathrm{~min}$. The gradient phase was set as follows: $0-8 \mathrm{~min}(100 \% \mathrm{~B})$, $8-15 \min (25 \% \mathrm{~A}, 75 \% \mathrm{~B}), 15-35 \mathrm{~min}(25 \% \mathrm{~A}, 75 \%$ B), $35-40 \min (100 \%$ B), $40-65 \min (100 \%$ B). Column temperature was maintained at $35^{\circ} \mathrm{C}$. The injection volume was $15 \mu \mathrm{L}$ of $5 \%(\mathrm{wt} / \mathrm{vol})$ sample in acetone. TAG peaks were identified by calculating the ECN value. Reference standards were triododecanoate, tripalmitin and trioleate.

Thermal profile analysis using Differential Scanning Calorimetry (DSC): A Perkin Elmer DSC 8000 (Waltham Massachusetts, USA) was used for examination of melting and crystallization of profile of the oil blend before and after transesterification. Nitrogen was used to purge the thermal analysis system at a flow rate of 20 $\mathrm{mL} / \mathrm{min}$. An empty hermetically sealed aluminium pan was used as reference. Sample of around 5.0-6.0 mg was weighed into an aluminium pan and sealed with cover. Prior to analysis of crystallization behavior, the sample was heated at $80^{\circ} \mathrm{C}$ for $5 \mathrm{~min}$ to melt all crystals and nuclei. It was then cooled to $-50^{\circ} \mathrm{C}$ at a rate of $5^{\circ} \mathrm{C} / \mathrm{min}$ and held at $-50^{\circ} \mathrm{C}$ for $10 \mathrm{~min}$ to fully crystallize the sample and obtain the crystallization thermogram. The sample was heated from $-50-80^{\circ} \mathrm{C}$ at a rate of $5^{\circ} \mathrm{C} / \mathrm{min}$ and held at $80^{\circ} \mathrm{C}$ for $10 \mathrm{~min}$ to record the melting thermogram (Lee et al., 2015). All measurements were performed with duplicate analysis.

\section{RESULTS AND DISCUSSION}

Immobilization of crude 1,3-regioselective ARM lipase: Crude ARM lipase was immobilized using simple 
Table 1: Immobilization of ARM lipase onto different supports

\begin{tabular}{lcc}
\hline Supports & Protein adsorbed (\%) & Activity $\left(\mu\right.$ mole $\mathrm{min}^{-1} / \mathrm{g}^{-1}$ or \\
$\mathrm{U} / \mathrm{g})$ & & 11.96 \\
Celite & 33.39 & 45.42 \\
Amberlite & 29.77 & 55.58 \\
Chitosan & 6.74 & \\
\hline
\end{tabular}

Total amount of protein offered for immobilization to $1 \mathrm{~g}$ of support 19 mg; lipase loading: $3016.67 \mathrm{U} / \mathrm{g}$ support

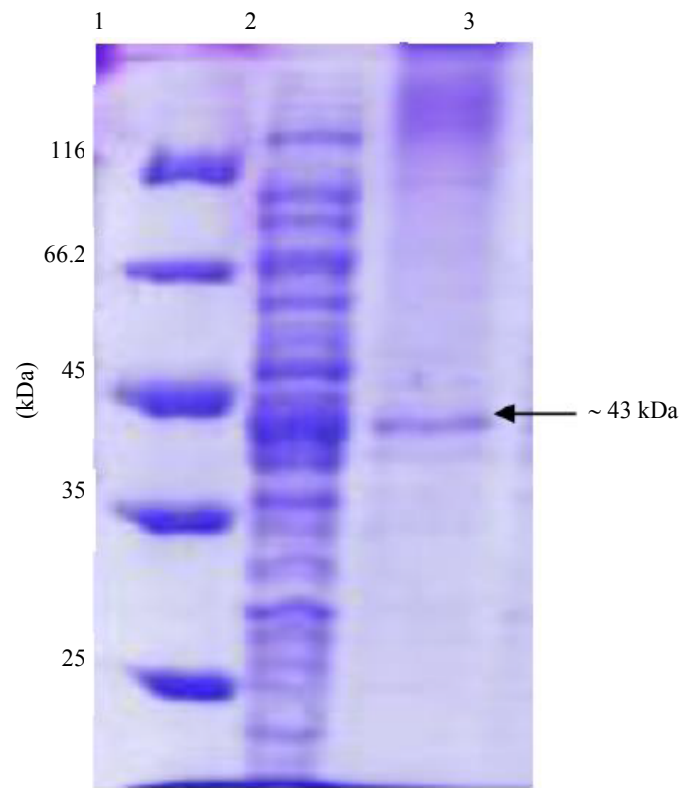

Fig. 1: SDS-PAGE analysis of thermostable 1,3 regioselective ARM lipase before and after immobilization on chitosan. Lane 1: Unstained Protein Molecular Weight Marker, Lane 2: crude enzyme, Lane 3: immobilized enzyme. Arrow indicates the target protein (ARM lipase) at approximately $43 \mathrm{kDa}$

physical adsorption method using several support matrices prior to enzymatic transesterification on both palm olein and palm kernel olein. Immobilization has been employed in many studies to improve enzyme stability (Nawani et al., 2006; Suescun et al., 2015) and is a strategy use to facilitate enzyme reuse, hence, reducing the processing cost (Adlercreutz, 2013). Screening for the suitable support for crude ARM lipase immobilization was made by comparing the protein amount adsorbed and activity performed by immobilized lipase on three different supports (celite, amberlite and chitosan). Table 1 depicts the percentage of protein adsorbed and lipolytic activity of the immobilized lipase.

Of the three supports, celite showed the highest percentage of adsorption ( $33.39 \%$ ), followed by amberlite and chitosan with 29.77 and $6.74 \%$, respectively. Conversely, result showed that the lipolytic activity of ARM lipase did not correspond to the percentage of adsorption. Chitosan-immobilized lipase displayed highest activity, $55.58 \mathrm{U} / \mathrm{g}$ as compared to amberlite and celite-immobilized lipase demonstrating a complete reversed trend to the adsorption percentage. Catalytic activity is one of the criteria evaluated in immobilized lipase preparation and achieving a high specific activity is extremely important to ensure the enzyme works at its maximum performance (Adlercreutz, 2013). Chitosan has been reported as a suitable support carrier for many lipases. Xie and Wang (2012) reported on the use of magnetic chitosan microsphere as a carrier for immobilization of commercial Candida rugosa lipase Lipase from Talaromyces thermophilus which was immobilized on chitosan pre-treated with glutaraldehyde, preserved its catalytic activity almost intact and offering maximum immobilization capacity (76 and $91 \%$, respectively) (Romdhane et al., 2013).

Further investigation on the bound protein was carried out by analyzing the SDS-PAGE profile. Interestingly, chitosan-immobilized lipase showed a significant reduction of impurities leaving almost a purified ARM lipase as clearly seen in Lane 2 (Fig. 1) as compared to its crude free enzyme (Lane 1). A distinct band with a correct size of ARM lipase (43-kDa) was observed on the gel. This result suggested that immobilization has remarkably facilitated the purification of ARM lipase. Besides, this qualitative analysis explains the high specific activity exhibited by chitosan-immobilized lipase due to the decline of impurities in the sample. Chitosan has been known for its excellent properties as a lipase support and is relatively economical (Foresti and Ferreira, 2007).

\section{Enzymatic synthesis of SLs from POo: PKO blend} Fatty acid composition: Fatty acid composition of $\mathrm{POo}$ : PKO $(50: 50 \mathrm{w} / \mathrm{w})$ before and after transesterification is shown in Table 2. Data revealed no significant difference in fatty acid composition at any reaction temperature which indicated that enzymatic modification did not change the overall fatty acid composition of the oil. The fatty acid proportion of the synthesized SLs is similar to that of the original blend, thus, confirming the efficacy of the reaction and displaying no degradation to physical factors such as heat and air during the process. This result is in agreement with other reported work where enzymatic interesterification causes the exchange of fatty acid composition within and between the TAGs, hence, the overall distribution remains unchanged (Reshma et al., 2008).

PKO is known to have high content of short and medium chain saturated fatty acids such as Caprylic (C8), Capric (C10) and lauric acid (C12) and contain low amount of unsaturated fatty acids, oleic 
J. Eng. Applied Sci., 14 (16): 5616-5626, 2019

Table 2: Fatty acid composition of POo:PKO $(50: 50 \mathrm{w} / \mathrm{w})$ before and after reaction at different temperatures

\begin{tabular}{|c|c|c|c|c|c|c|}
\hline \multirow[b]{2}{*}{ Types of fatty acid } & \multicolumn{6}{|c|}{ Amount (\%) } \\
\hline & Blend ${ }^{2}$ & $35^{\circ} \mathrm{C}$ & $40^{\circ} \mathrm{C}$ & $45^{\circ} \mathrm{C}$ & $50^{\circ} \mathrm{C}$ & $55^{\circ} \mathrm{C}$ \\
\hline $\mathrm{C} 8$ & $1.357 \pm 0.00$ & $1.374 \pm 0.06$ & $1.449 \pm 0.01$ & $1.308 \pm 0.06$ & $1.496 \pm 0.11$ & $1.495 \pm 0.01$ \\
\hline $\mathrm{C} 10$ & $1.489 \pm 0.02$ & $1.434 \pm 0.04$ & $1.435 \pm 0.01$ & $1.489 \pm 0.10$ & $1.575 \pm 0.07$ & $1.429 \pm 0.00$ \\
\hline $\mathrm{C} 12$ & $23.51 \pm 0.01$ & $23.64 \pm 0.01$ & $23.43 \pm 0.04$ & $23.29 \pm 0.03$ & $23.34 \pm 0.04$ & $23.44 \pm 0.03$ \\
\hline C14 & $8.618 \pm 0.01$ & $8.722 \pm 0.01$ & $8.739 \pm 0.03$ & $8.775 \pm 0.05$ & $8.623 \pm 0.01$ & $8.767 \pm 0.05$ \\
\hline C16 & $24.49 \pm 0.01$ & $24.51 \pm 0.00$ & $24.46 \pm 0.05$ & $24.59 \pm 0.01$ & $24.25 \pm 0.00$ & $24.44 \pm 0.03$ \\
\hline $\mathrm{C} 18$ & $4.047 \pm 0.00$ & $4.092 \pm 0.03$ & $4.056 \pm 0.17$ & $4.118 \pm 0.05$ & $4.021 \pm 0.03$ & $4.094 \pm 0.10$ \\
\hline C18:1 & $29.88 \pm 0.03$ & $29.64 \pm 0.02$ & $29.76 \pm 0.10$ & $29.75 \pm 0.04$ & $29.28 \pm 0.03$ & $29.68 \pm 0.01$ \\
\hline C18:2 & $6.618 \pm 0.01$ & $6.593 \pm 0.02$ & $6.667 \pm 0.06$ & $6.672 \pm 0.01$ & $6.564 \pm 0.01$ & $6.662 \pm 0.01$ \\
\hline SFA & 63.51 & 63.77 & 63.57 & 63.57 & 63.31 & 63.67 \\
\hline MUFA/PUFA & 36.49 & 36.23 & 36.43 & 36.42 & 35.84 & 36.34 \\
\hline
\end{tabular}

Types of fatty acid SFA = Saturated Fatty Acid, MUFA/PUFA= Mono/Polyunsaturated Fatty Acid. Each value in the table represents the mean \pm standard deviation; Types of oils. Blend $=$ POo: PKO blend without enzyme added (control)

(C18:1) and linoleic (C18:2). To improve the quality and usefulness of $\mathrm{PKO}$, the oil is often interesterified with other oil such as palm stearin for margarine production. In this research, blending of $\mathrm{PKO}$ with $\mathrm{POo}$ was seen to increase the Monounsaturated Fatty Acid (MUFA) and Polyunsaturated Aatty Acid (PUFA) content, since, the latter oil contains approximately $41.5 \%$ of $\mathrm{C} 18: 1$ (oleic) and $11.6 \%$ of $\mathrm{C} 18: 2$ (linoleic). High MUFA (in this case C18:1) diet is advantageous compared to PUFA (C18:2). The oil blend which composed of almost an equal percentage of lauric acid $(23.51 \%)$ and oleic acid (29.88\%) could be exploited for the production of more nutritious and wholesome oil.

Acylglycerol composition: Fats and oils have complex composition of acylglycerols (TAG, DAG, MAG and free fatty acids). Precise identification and quantification of the acylglycerols are vital to correlate the effect of modification on food lipid physical properties such as solid fat content and melting point (Neff et al., 2002; Lee et al., 2015). Lipase-mediated transesterification can be evaluated by looking at the changes in TAG concentration or formation of new TAGs. Apart from the changes in TAG concentration, the percentage of Free Fatty Acids (FFA\%) is equally important to monitor the transesterification reaction, since, it occurs alongside hydrolysis (Ghazali et al., 1995; Ming et al., 1999).

Using HPLC, TAG species was determined according to, retention time and Equivalent Carbon Number (ECN). The TAG composition of oil blend PKO: POo was evaluated before and after the transesterification reaction up to $10 \mathrm{~h}$. Based on HPLC chromatogram (Fig. 2a), the initial composition of the oil blend consisted of several major TAGs of PKO; CaLaLa, LaLaLa, LaLaM, LaLaM/LaLaP and POo; OOO, POO and PPO. The proportion of POo and PKO has linear correlation with TAG composition in the blend (Lida et al., 2002).

Changes in TAGs composition were more substantial at higher temperature and longer incubation time when the oil blend was subjected to transesterification reaction using ARM lipase. Figure $2 b$ shows the TAG species observed after $10 \mathrm{~h}$ of the enzymatic reaction carried out at $55^{\circ} \mathrm{C}$. The yield of LaLaLa, CaLaLa, LaLaM, $\mathrm{LaLaM} / \mathrm{LaLaP}, \mathrm{LaLaO}, \mathrm{POO}$ and $\mathrm{PPO}$ dominated the TAG species detected. Several MLCT species can be seen after the enzymatic reaction including $\mathrm{LaLaO}, \mathrm{LaLaP}$, LaLaM suggesting that the transesterification has randomized and restructured the FFA composition of the reacted oil blend. The incorporation of palmitic acid (C16) and oleic acid (C18:1) into the major PKO fatty acid lauric acid (C12) generates a good blend with better functionality. Additionally, a new peak of DAG was also spotted after the enzymatic reaction. DAG is one of the important components in healthy food oil.

Effect of reaction time: Further interpretation of HPLC results was carried out to observe the composition of TAGs at two profound variables temperature $\left(35-55^{\circ} \mathrm{C}\right)$ and reaction time $(0-10 \mathrm{~h})$. As shown in Fig. 3, MLCT production was influenced by the reaction time where prolonged enzymatic reaction $(6-10 \mathrm{~h})$ improved the product yield, especially at $55^{\circ} \mathrm{C}$. At lower reaction temperature $\left(40,45,50^{\circ} \mathrm{C}\right)$, almost a consistent yield of MLCT with marginal increment was observed throughout the total reaction time. The $\mathrm{MLCT}$ which include $\mathrm{LaLaO}$, $\mathrm{LaLaM} / \mathrm{LaLaP}, \mathrm{LaOL} / \mathrm{LaPL}$, LaMO, LaMP and LaPP is a type of structured lipid consisting of both Medium Chain Fatty Acids (MCFA) and Long Chain Fatty Acids (LCFA) in a TAG. MCFA exhibits greater value than the LCFA due to its higher solubility and smaller molecular size which allows the absorption, transport and metabolism much easier than the latter form of fatty acids (Hamam, 2013). Nevertheless, the incorporation of LCFA will provide the essential fatty acid to the body (Lee et al., 2015).

MLCT reached its maximum yield within $4-7 \mathrm{~h}$ and further increase in reaction time has no effect to the yield except for reaction conducted at $55^{\circ} \mathrm{C}$. This signifies that the transesterification has reached its steady state within 

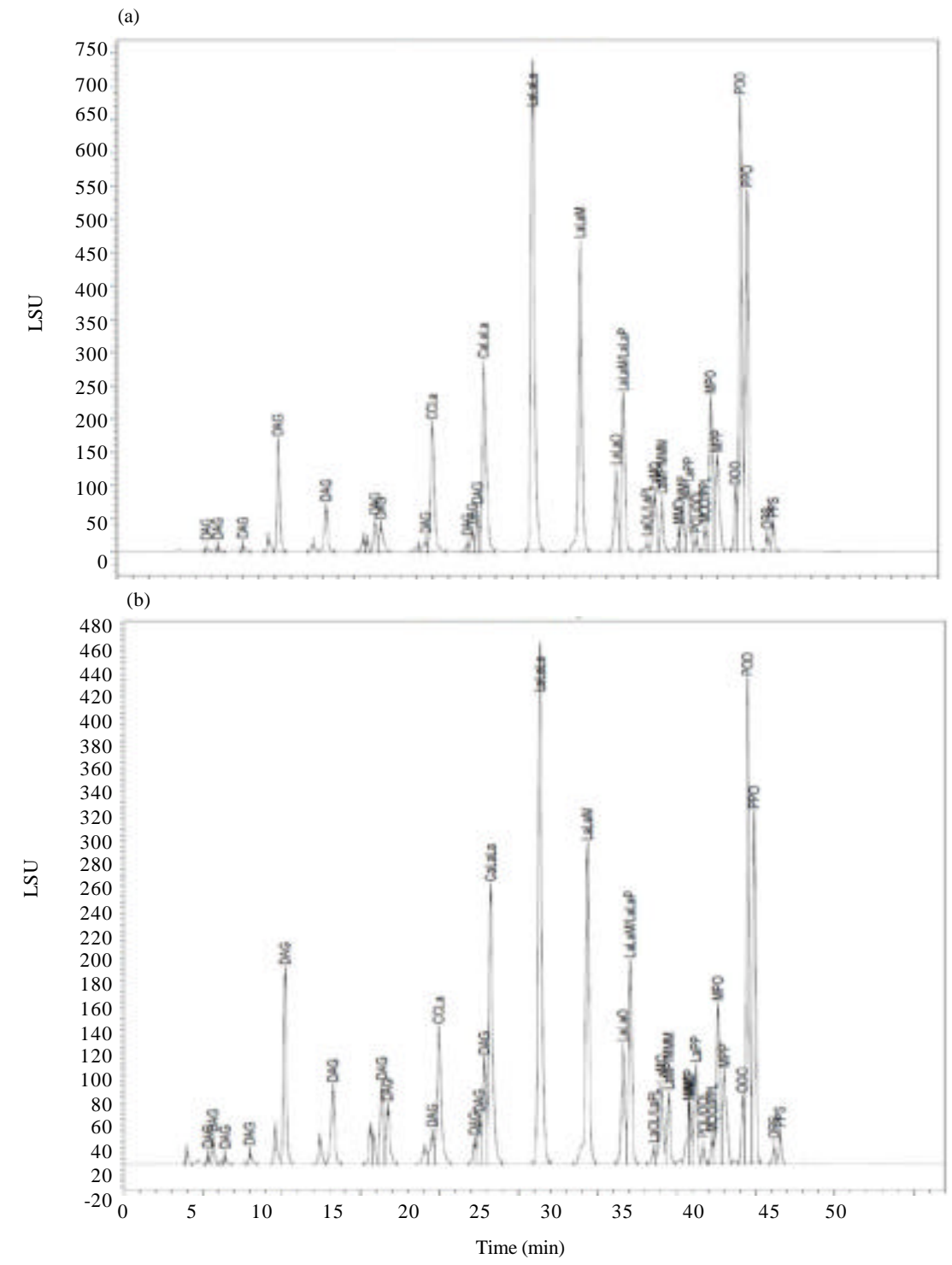

Fig. 2: HPLC chromatogram showing POo: $\mathrm{PKO}(50: 50 \mathrm{w} / \mathrm{w})$ before and after enzymatic transesterification reaction; a) Before enzymatic reaction and b) After enzymatic transesterification reaction at $55^{\circ} \mathrm{C}, 10 \mathrm{~h}$. MAG/DAG $=$ mono and diacylglycerol, $\mathrm{C}=$ Caprylic, $\mathrm{Ca}=$ Capric, $\mathrm{La}=$ Lauric, $\mathrm{M}=$ Myristic, $\mathrm{P}=$ Palmitic, $\mathrm{O}=$ Oleic, $\mathrm{L}=$ Linoleic

the period. Meanwhile, the concentration of DAG, a by product of hydrolysis was also found to dramatically increase with the increasing of reaction time starting from 2 up to 8 h of reaction. This could be due to the presence of water trace after prolonged reaction. Several methods of producing DAG have been reported including partial hydrolysis of TAG, esterification of FFA or its derivatives with glycerol or a combination of both methods (Guo and Sun, 2007). Watanabe et al. (2003) reported on the synthesis of $84 \% \quad 1,3$ DAG using immobilized 1,3regiospecific lipase Lipozyme RM1M. The FFA/MAG yield on the other hand, showed an increasing trend throughout the reaction time but of lower amount compared to the former two products. A slight deviation from the general trends was observed at $35^{\circ} \mathrm{C}$ (during 9$10 \mathrm{~h}), 45^{\circ} \mathrm{C}$ (during $9-10 \mathrm{~h}$ ) and $50^{\circ} \mathrm{C}$ (during $10 \mathrm{~h}$ ), suggesting that FFAs liberated from previous hours could be re-esterified to form TAG in the extended time (data not shown). This is in agreement to a report suggesting that prolong reaction time will increase the formation of by products (free fatty acids and glycerol) and cause randomization of fatty acids in the glycerol backbone (Lee et al., 2015). In food industry, oil with these by products need to be subjected with additional refining processes and hence, the cost of production will increase. 

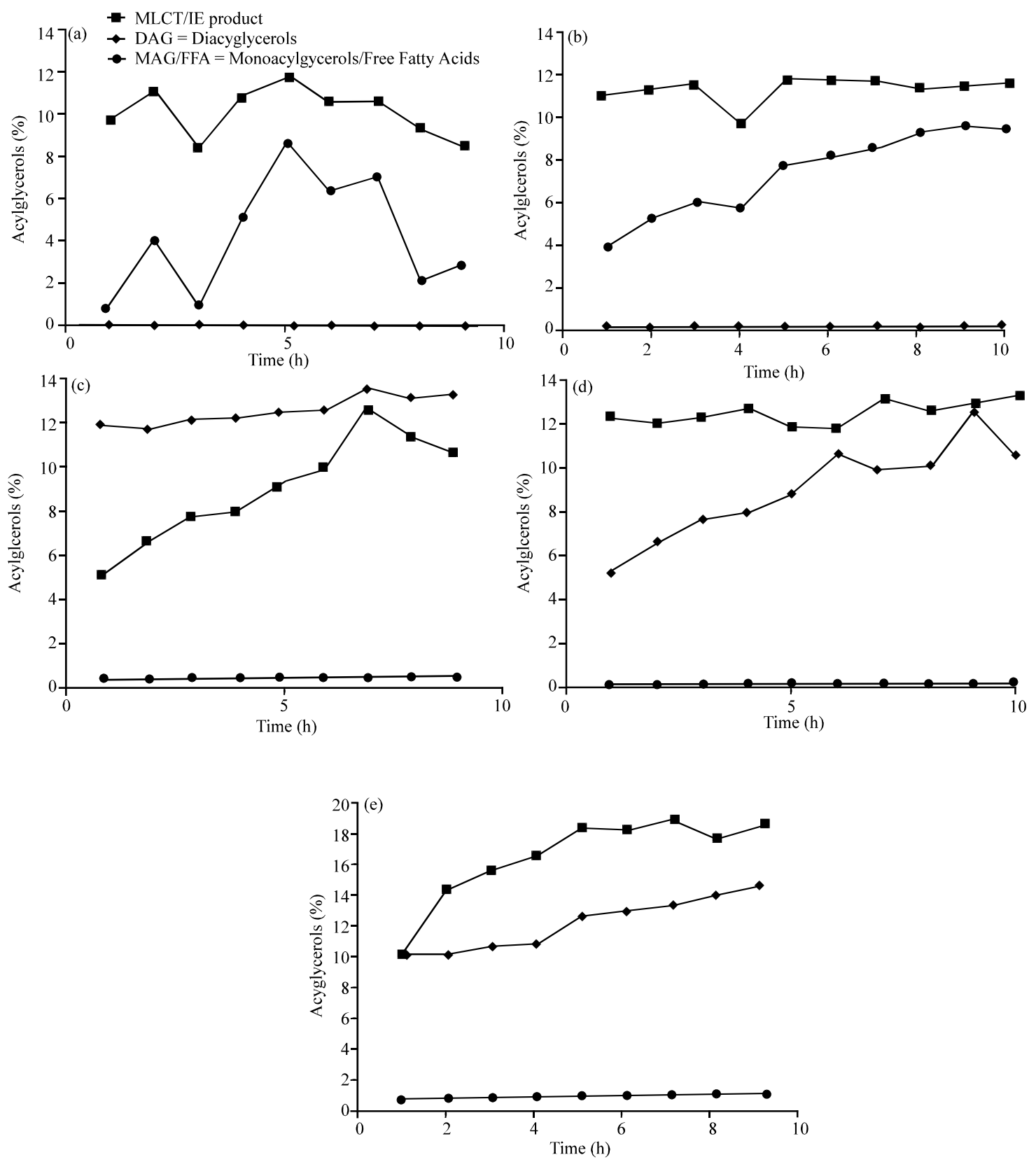

Fig. 3: Acylglycerol composition (\%) of the oil blend at various reaction temperatures; a) $35^{\circ} \mathrm{C}$; b) $40^{\circ} \mathrm{C}$; c) $45^{\circ} \mathrm{C}$; d) $\left.50^{\circ} \mathrm{C}^{\circ}\right) 55^{\circ} \mathrm{C}$ and e(- - MLCT/IE product $=$ Medium-and Long-Chain Triacylglycerols/interesterification product, (-^) DAG = Diacylglycerols, (-^) MAG/FFA= Monoacylglycerols/Free Fatty Acids

Effect of reaction temperature: For temperature variable, oil blend which has been enzymatically transesterified at $55^{\circ} \mathrm{C}$ showed a significant increase in MLCT yield, especially, at $10 \mathrm{~h}$ with $14.5 \%$ (Fig. 3e). In parallel with that of MLCT, the DAG yield was also increased. The yield of MLCT and DAG indicates that 1,3-specific ARM lipase has successfully promoted fatty acids redistribution of the reacted blend, producing 1,2- or 2,3- diacylglycerols (1,2-or 2,3-DAGs). MLCT and DAG are products of enzymatic transesterification and partial hydrolysis, respectively. The use of 1,3-specific lipase in the reaction could be advantageous in incorporating Free Fatty Acids (FFA) present in the reaction medium to the sn-1 and sn-3 positions. Wang et al. (2012) 
suggested that the metabolic and physical properties of SLs are influenced by the regiospecific positions of FA in the TAG molecules. Many 1,3 specific lipases have been reported in the production of Sls such as 1,3 Rhizomucor miehei lipase which was used in the acidolysis of sunflower oil and palmitic-stearic acid mixture (Palla and Carrin, 2014). Lipase NRRLY-7723 from Pseudomonas lynferdii exhibited 1,3regiosepecificity toward the TAG of borage oil (Kim et al., 2010).

Figure $3 \mathrm{a}$, at lower reaction temperature, $35^{\circ} \mathrm{C}$, the MLCT concentration is relatively low demonstrating a poor catalytic activity of ARM lipase in catalyzing the transesterification of the oil blend. The synthesis of MLCT, however, gradually improved as the reaction temperature increased from $40-55^{\circ} \mathrm{C}$ with $5^{\circ} \mathrm{C}$ interval (Fig. $3 \mathrm{~b}$-d). The results clearly depicted that reaction carried out at $55^{\circ} \mathrm{C}$ exhibited the highest conversion of TAG into MLCT and DAG. Apart from MLCT, DAG oil has been regarded as novel functional lipid (Xu et al., 2016). The dramatic increase in the reaction rate is suggested by the enhancement of lipolytic activity at high temperature. ARM lipase has been earlier reported to exhibit optimum temperature at $60^{\circ} \mathrm{C}$ (Ebrahimpour et al., 2011). Interestingly, the enzyme displayed excellent stability even when subjected to $10 \mathrm{~h}$ reaction at $55^{\circ} \mathrm{C}$. Similar finding on $1,3-\mathrm{DG}$ production was also reported at temperature between 45 and $55^{\circ} \mathrm{C}$ using commercial lipase, Novozym 435. Temperature was described to play significant role in acyl migration (Hamam, 2013). Apart from the intrinsic stability of ARM lipase at elevated temperature, immobilization may have synergistically improved its functional stability and performance.

Melting and crystallization behavior: The thermal profile of fats and oils is dependent on the distribution of TAG species. Lacking of specific melting point as PO consists of high and low melting point Triacylglycerols (TAGs), a temperature range where the oil melts is important to depict the re-arrangement of TAGs in the oil (Zhang et al., 2013). Using Differential Scanning Calorimetry (DSC) as an analytical tool to study the physical properties of $\mathrm{POo}: \mathrm{PKO}$ blend, melting and crystallization behaviors were analyzed at different temperature transition during cooling and heating.

Several parameters including the onset temperature, enthalpy (heat flow status) and end set temperature were measured during the cooling stage (crystallization) of oil blend. The crystallization profile is described by the beginning of fat crystal formation due to reorganization of molecules in the presence of high saturated FA and the development of aggregation and molecules compaction (Saadi et al., 2012). Figure 4 shows the crystallization and melting curve of POo:PKO blend at 50: $50(\mathrm{w} / \mathrm{w})$ measured before and after the enzymatic reaction. The crystallization behavior of the oil blend was somewhat different after $10 \mathrm{~h}$ of enzymatic reaction as compared to the blend prior to reaction. Initially, DSC thermogram revealed two crystallization peaks with one sharp peak (Fig. 4a) at temperature range of $0.99^{\circ} \mathrm{C} \pm 0.33$ and $7.33^{\circ} \mathrm{C} \pm 0.21$. Although, similar pattern of crystallization profile was observed at different reaction temperatures, a marginal shift of the crystallization peak was detected as the temperature increased. At the highest reaction temperature, $55^{\circ} \mathrm{C}$ the exothermic peak was visible at $4.1^{\circ} \mathrm{C} \pm 0.31$ and $9.45^{\circ} \mathrm{C} \pm 0.36$. This could be an indication of redistribution of FA in the oil blend, hence, resulting in a slight difference in the physical behavior as compared to the initial blend. Such observation is often seen in oil samples wherethey display multiple exotherms when cooled in DSC (Tan and Man, 2002). This is true for the case of Poo: PKO blend when not subjected to enzymatic transesterification where a single exothermic peak is associated with another peak with small temperature difference. Crystallization temperature and composition of the blend has been reported to significantly influence the mechanism of crystallization (Rashid et al., 2012).

As for the melting profile, the curve was found more broad and irregular than the crystallization curves. Frederick et al. (2008) suggested that the amount of crystallized fat and the presence of polymorphic transitions are depicted in the melting profiles. Prior to enzymatic transesterification reaction, the original blend melted in one broad fraction associated with a shoulder where the endothermic peak was detected at $19.38^{\circ} \mathrm{C}$ with $\mathrm{T}_{\text {on }} 1.38^{\circ} \mathrm{C}$ and $\mathrm{T}_{\text {off }} 25.10^{\circ} \mathrm{C}$. The broadening effect may be due to broad TAG distribution in the oil blend where it consists of high and low melting point TAGs (Ebrahimpour et al., 2011). As can be seen in Fig. 4b when the temperature increased, the shoulder began to disappear leaving a smooth broad peak with a slight shift towards lower temperature except for reaction conducted at $40^{\circ} \mathrm{C}$. At $55^{\circ} \mathrm{C}$, the endothermic peak was detected in a temperature range around 0.29 and $22.88^{\circ} \mathrm{C}$.

The shift in melting temperature of the oil blend was probably influenced by the altered percentage of saturated and unsaturated fatty acid as previously described in Table 2 and Fig. 2. High melting peak is often attributed by the presence of SSS (SaturatedSaturated-Saturated) while low melting peak corresponds to UUU (Unsaturated-Unsaturated Unsaturated) content of TAG species. Meanwhile the melting peak for mixture of saturated and unsaturated fatty acid lies between the two extremes. The broad melting peak observed in the original blend indicates the 

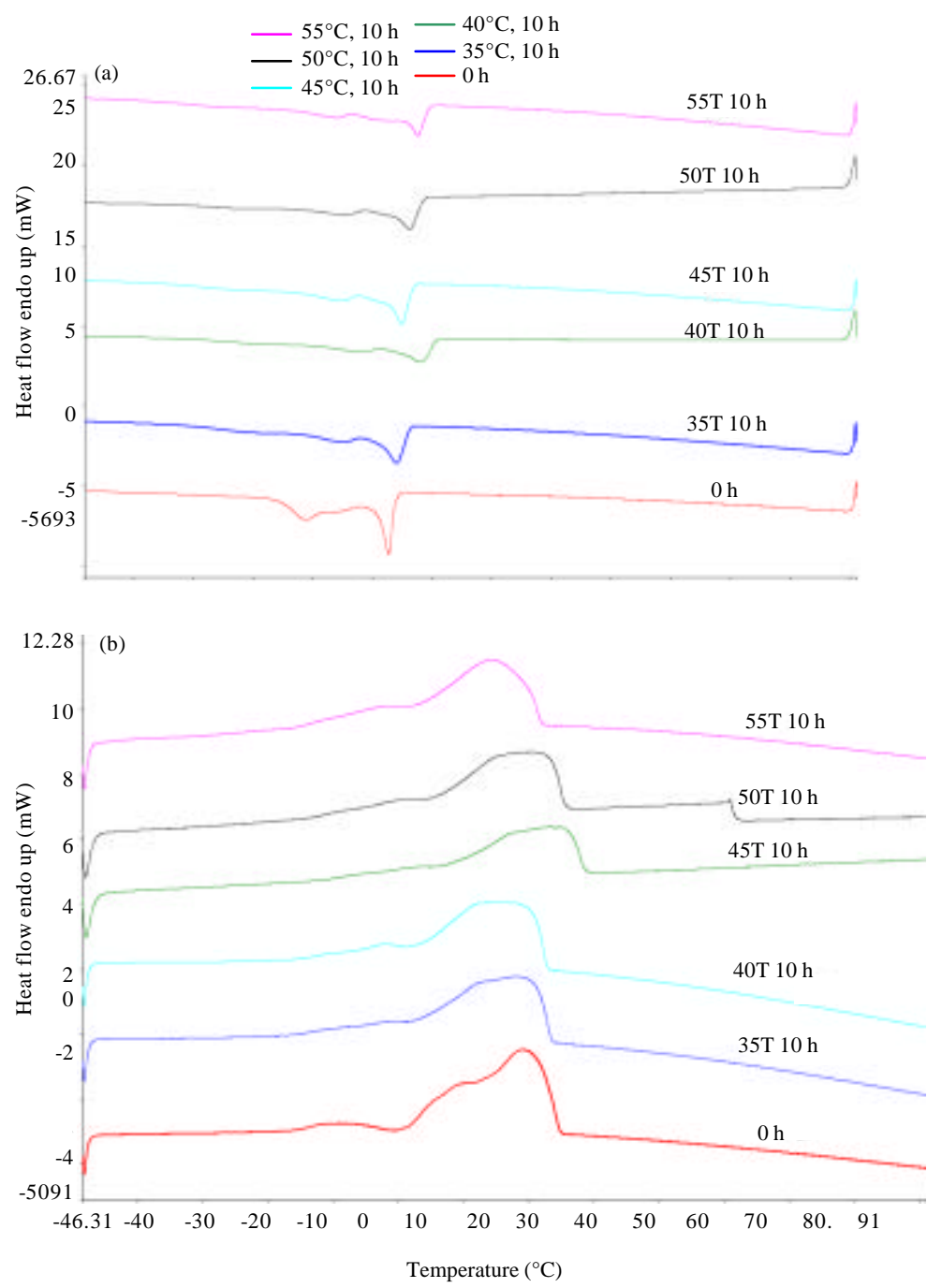

Fig. 4: DSC thermograms of POO: $\mathrm{PKO}$ blend $(50: 50 \mathrm{w} / \mathrm{w})$ before and after enzymatic reaction at various temperatures, $35-55^{\circ} \mathrm{C}$ with $5^{\circ} \mathrm{C}$ interval; a) Cooling curves and b) Melting curves. Lines are indicated as follows: $(-) 55^{\circ} \mathrm{C}$, $10 \mathrm{~h} ;(-) 50^{\circ} \mathrm{C}, 10 \mathrm{~h} ;(-) 45^{\circ} \mathrm{C}, 10 \mathrm{~h} ;(-) 40^{\circ} \mathrm{C}, 10 \mathrm{~h} ;(-) 35^{\circ} \mathrm{C}, 10 \mathrm{~h} ;(-) 0 \mathrm{~h}=$ before enzymatic transesterification

presence of SSS (LaLaLa, LaLaM, LaLaP), SSU $(\mathrm{LaLaO})$, SUU (POO) and SSU (PPO) which occur in various quantity. As the enzymatic reaction took place, the yield of $\mathrm{LaLaO}$, LaLaP and LaLaM became more significant, hence, varying the melting behavior.

\section{CONCLUSION}

In this research, we aim to synthesize new structured lipids deriving from oil blend of palm oil derivatives palm olein and palm kernel olein by lipase-catalyzed transesterification using locally isolated, immobilized thermostable 1,3-regioselective lipase from Geobacillus sp. strain ARM (designated as ARM lipase). To our best knowledge, literature reporting on blending of the palm oil and palm kernel oil derivatives is relatively limited.
Oil blending is one of the effective ways to produce SLs for specific utilizations. Additionally, discovery of new local microbial lipase capable of synthesizing SLs at high temperature may contribute to development of competent biocatalyst for industrial exploitation.

\section{ABBREVIATIONS}

$\mathrm{SL} \quad=$ Structured lipid

$\mathrm{POo}=$ Palm Olein

$\mathrm{PKO}=$ Palm Kernel Olein

MCFA $=$ Medium Chain Fatty Acid

LCFA $=$ Long Chain Fatty Acid

MLCT $=$ Medium and Long Chain Triacylglyceride

$\mathrm{DAG}=$ Diacylglyceride

$\mathrm{FA} \quad=$ Fatty Acid

$\mathrm{TAG}=$ Triacylglycerol 


\section{ACKNOWLEDGEMENT}

This project was financially supported by Ministary of Science, Technology and Innovation (MOSTI), Malaysia (02-01-04-SF2460).

\section{REFRENCES}

Adlercreutz, P., 2013. Immobilisation and application of lipases in organic media. Chem. Soc. Rev., 42: 6406-6436.

Branco, R.V., E. Gutarra, M. Limoeiro, D.M.G. Freire and R.V. Almeida, 2010. Immobilization and characterization of a recombinant therm ostable lipase (Pf2001) from Pyrococcus furiosus on supports with different degrees of hydrophobicity. Enzyme Res., 2010: 1-8.

Cai, H., Y. Li, M. Zhao, G. Fu and J. Lai et al., 2015. Immobilization, regiospecificity characterization and application of Aspergillus oryzae lipase in the enzymatic synthesis of the structured lipid 1,3-dioleoyl-2-palmitoylglycerol. PloS One, 10: $1-19$.

Cao, Y., S. Qi, Y. Zhang, X. Wang and B. Yang et al., 2013. Synthesis of structured lipids by lipase-catalyzed interesterification of triacetin with camellia oil methyl esters and preliminary evaluation of their plasma lipid-lowering effect in mice. Mol., 18: 3733-3744.

Chen, M.L., S.R. Vali, J.Y. Lin and Y.H. Ju, 2004. Synthesis of the structured lipid 1,3-dioleoy 1-2palmitoylglycerol from palm oil. J. Am. Oil Chem. Soc., 81: 525-532.

Ebrahimpour, A., R.N.Z.R.A. Rahman, M. Basri and A.B. Salleh, 2011. High level expression and characterization of a novel thermostable, organic solvent tolerant, 1,3-regioselective lipase from Geobacillus sp. strain ARM. Bioresour. Technol., 102: 6972-6981.

Foresti, M.L. and M.L. Ferreira, 2007. Chitosan-immobilized lipases for the catalysis of fatty acid esterifications. Enzyme Microb. Technol., 40: 769-777.

Fredrick, E., I. Foubert, J. Van De Sype and K. Dewettinck, 2008. Influence of monoglycerides on the crystallization behavior of palm oil. Cryst. Growth Des., 8: 1833-1839.

Ghazali, H.M., S. Hamidah and Y.B. Che Man, 1995. Enzymatic transesterification of palm olein with nonspecific and 1,3-specific lipases. J. Am. Oil Chem. Soc., 72: 633-639.

Guo, Z. and Y. Sun, 2007. Solvent-free production of 1,3-diglyceride of CLA: Strategy consideration and protocol design. Food Chem., 100: 1076-1084.
Hamam, F., 2013. Specialty lipids in health and disease. Food Nutr. Sci., 4: 63-70.

Khodadadi, M., S. Aziz, R. St-Louis and S. Kermasha, 2013. Lipase-catalyzed synthesis and characterization of flaxseed oil-based structured lipids. J. Funct. Foods, 5: 424-433.

Kim, H.R., C.T. Hou, K.T. Lee, B.H. Kim and I.H. Kim, 2010. Enzymatic synthesis of structured lipids using a novel cold-active lipase from Pichia lynferdii NRRL Y-7723. Food Chem., 122: 846-849.

Koh, S.P., K. Long, C.P. Tan, M.S.A. Yusoff and O.M. Lai et al., 2011. The use of enzymatically synthesized Medium-and Long-Chain Triacylglycerols (MLCT) oil blends in food application. Intl. Food Res. J., 18: 355-365.

Kwon, D.Y. and J.S. Rhee, 1986. A simple and rapid colorimetric method for determination of free fatty acids for lipase assay. J. Am. Oil Chem. Soc., 63: $89-92$

Lee, Y.Y., T.K. Tang, E.T. Phuah, N.A. Ab Karim and S.M.M. Alwi et al., 2015. Palm-based Medium-and-Long-Chain Triacylglycerol (P-MLCT): Production via enzymatic interesterification and optimization using Response Surface Methodology (RSM). J. Food Sci. Technol., 52: 685-696.

Lida, H.N., K. Sundram, W.L. Siew, A. Aminah and S. Mamot, 2002. TAG composition and solid fat content of palm oil, sunflower oil and palm kernel olein belends before and after chemical interesterification. J. Am. Oil Chem. Soc., 79: 1137-1144.

Ming, L.O., H.M. Ghazali and C.C. Let, 1999. Use of enzymatic transesterified palm stearin-sunflower oil blends in the preparation of table margarine formulation. Food Chem., 64: 83-88.

Nawani, N., R. Singh and J. Kaur, 2006. Immobilization and stability studies of a lipase from thermophilic Bacillus sp. The effect of a process parameters on immobilization of enzyme. Elect. J. Biotechnol., 90: 563-565.

Neff, W.E., W.C. Byrdwell, K.R. Steidley, G.R. List and G. Snowder, 2002. Triacylglycerol structure of animal tallows, potential food formulation fats by high performance liquid chromatography coupled with mass spectrometry. J. Liq. Chromatogr. Relat. Technol., 25: $985-998$.

O'fallon, J.V., J.R. Busboom, M.L. Nelson and C.T. Gaskins, 2007. A direct method for fatty acid methyl ester synthesis: Application to wet meat tissues, oils and feedstuffs. J. Anim. Sci., 85: 1511-1521.

Palla, C.A. and M.E. Carrin, 2014. Kinetics modeling of the acidolysis with immobilized Rhizomucor miehei lipases for production of structured lipids from sunflower oil. Biochem. Eng. J., 90: 184-194. 
Rahman, M.B.A., U.H. Zaidan, M. Basri, M.Z. Hussein and R.N.Z.R.A. Rahman et al., 2008. Enzymatic synthesis of methyl adipate ester using lipase from Candida nugosa immobilised on $\mathrm{Mg}, \mathrm{Zn}$ and $\mathrm{Ni}$ of Layered Double Hydroxides (LDHs). J. Mol. Catal. B. Enzym., 50: 33-39.

Rashid, N.A., C.C. Let, C.C. Seng and Z. Omar, 2012. Crystallisation kinetics of palm stearin, palm kernel olein and their blends. LWT. Food Sci. Technol., 46: 571-573.

Reshma, M.V., S.S. Saritha, C. Balachandran and C. Arumughan, 2008. Lipase catalyzed interesterification of palm stearin and rice bran oil blends for preparation of zero trans shortening with bioactive phytochemicals. Bioresour. Technol., 99: 5011-5019.

Romdhane, I.B.B., Z.B. Romdhane, M. Bouzid, A. Gargouri and H. Belghith, 2013. Application of a chitosan-immobilized Talaromyces thermophilus lipase to a batch biodiesel production from waste frying oils. Appl. Biochem. Biotechnol., 171: 1986-2002.

Saadi, S., A.A. Ariffin, H.M. Ghazali, M.S. Miskandar and H.C. Boo et al., 2012. Application of Differential Scanning Calorimetry (DSC), HPLC and pNMR for interpretation primary crystallisation caused by combined low and high melting TAGs. Food Chem., 132: 603-612.

Suescun, A., N. Rueda, J.C. Dos Santos, J.J. Castillo and C. Ortiz et al., 2015. Immobilization of lipases on glyoxyl-octyl supports: Improved stability and reactivation strategies. Process Biochem., 50: 1211-1217.
Tan, C.P. and Y.B.C. Man, 2002. Differential scanning calorimetric analysis of palm oil, palm oil based products and coconut oil: Effects of scanning rate variation. Food Chem., 76: 89-102.

Wang, Y., L. Xia, X. Xu, L. Xie and Z. Duan, 2012. Lipase-catalyzed acidolysis of canola oil with caprylic acid to produce medium-, long-and medium-chain-type structured lipids. Food Bioprod. Process., 90: 707-712.

Watanabe, T., M. Shimizu, M. Sugiura, M. Sato and J. Kohori et al., 2003. Optimization of reaction conditions for the production of DAG using immobilized 1,3-regiospecific lipase lipozyme RMIM. J. Am. Oil Chem. Soc., 80: 1201-1207.

Xie, W. and J. Wang, 2012. Immobilized lipase on magnetic chitosan microspheres for transesterification of soybean oil. Biomass Bioenergy, 36: 373-380.

Xu, Y., X. Zhao, Q. Wang, Z. Peng and C. Dong, 2016. Thermal profiles, crystallization behaviors and microstructure of diacylglycerol-enriched palm oil blends with diacylglycerol-enriched palm olein. Food Chem., 202: 364-372.

Zhang, X., L. Li, H. Xie, Z. Liang and J. Su et al., 2013. Comparative analysis of thermal behavior, isothermal crystallization kinetics and polymorphism of palm oil fractions. Mol., 18: 1036-1052. 